\title{
An Annotation Paradigm: The Social Hyperlink
}

\author{
Thomas Ciszek \\ Xin Fu \\ School of Information and Library Science, University of North Carolina at Chapel Hill, CB\# 3360, 100 Manning \\ Hall, Chapel Hill, NC 27599-3360 Email: \{tommy, fu\}@email.unc.edu
}

\begin{abstract}
We seek to understand how hyperlinks act as agents of annotation and play a fundamental role in how we seek and interpret information on the Internet. Recognizing that the hyperlink is a form of latent human annotation, we emphasize the significance of hyperlinking to establish authority. Furthermore, we assess the evolution of hyperlink taxonomies, acknowledging that as annotations, hyperlinks have developed into socio-cognitive entities. In the second generation of the Internet (particularly the Blogosphere), we seek to confirm the existence of social motivations for hyperlinking; thus substantiating the idea of a social hyperlink as a phenomenon prerequisite to the development of a social-linking theory.
\end{abstract}

\section{Background}

Many questions remain about how to best design the linked and diverse resources of the Web. Annotations are the basis on which the web grows, linking site to site through hyperlinks anchored on any of a variety of media. Links of this sort form associative relationships between nodes of information. A collection of hyperlinks as annotations on text or media, convey the descriptive organizational structure of an information model. The authors of the webpage shape the structure and characteristics of this model. How do hyperlinks act as annotation agents? Why they are fundamental in the seeking and interpreting of information in a web context?

\section{Prior Research}

Previous research suggests that the reasons for linking are an important facet in understanding the quality of a document. To further the validity of webometric research, it is necessary to study the underlying factors - the reasons for linking. Often compared with the reasons for citing (particularly in scholarly electronic publications), there are similar rationalizations. Influenced by internal (personal, behavioral, psychological) and external forces (technological skill, cultural, organizational, etc.), author motivations for citation and hyperlinking are social constructs. Difficulty arises with attempts to reconcile these social elements to optimize human information interaction. Moreover, the continuously increasing universe of web pages and the rapid evolution of the Internet hamper efforts to frame a hyperlink taxonomy.

\section{Hypertext Taxonomies}

There are several studies on making hyperlinks in a hypertext environment, such as DeRose's (1989); but as Haas and Grams (1998) points out, "a hypertext system is generally created with a single purpose or theme" and "there is generally a sense of unity and similarity of style" throughout a hypertext system, which is not true for the rich media of a Web environment. DeRose's taxonomy of link types is divided into two major classes, extensional and intensional. The extensional links must be defined and stored individually; they are based on the author's suggested use of the hypertext. Intensional links can be automatically inferred. Haas and Grams (1998), develop their link type classification structure from an examination of the setting in which the anchor was placed, the anchor itself, and the apparent reason that a reader might follow the link. The four link types identified are: navigation, expansion, resource, and miscellaneous.

\section{Hyperlink Taxonomies for the Web}

There are also some proposals for Web link taxonomies, but most of them cater to specific domains, such as academic (Thelwall 2003), or educational pages (Chu, 2005), or to one genre, such as electronic articles (Kim, 2000). As Chu (2005) indicates, "research on why Web links are created has just begun.” Thelwall (2003) postulates four new types of motivations. The term "ownership" is coined for links acknowledging authorship or co-authorship of a resource, "social" for links with a primarily social reinforcement role, "general navigational" for those with a general information navigation function and "gratuitous" for those that serve no communication function at all. Thelwall claims that compared to citer motivations, hyperlinks are relatively trivial and that instead of being primarily socio-cognitive, none of the hyperlinking motivations is cognitive and the gratuitous "does not seem to have a primarily social motivation."

\section{Goals}

There is increasing evidence with which to oppose Thelwall's argument. Hyperlinking is a socially motivated action and is truly a cognitive act. Hyperlinks play a profound role in the development of Weblogs. The Pew Internet \& American Life Project's report on blogging (2005), states that Weblogs (blogs) have become "a key part of online culture.” As such, they provide a platform from which to research the social functionality of 
hyperlinking. Working from within a synthesized taxonomic framework for evaluation and classification of hyperlinks, we hypothesize that hyperlinks have evolved beyond being citation knowledge tools to become sociallinking mechanisms [See Figure 1].

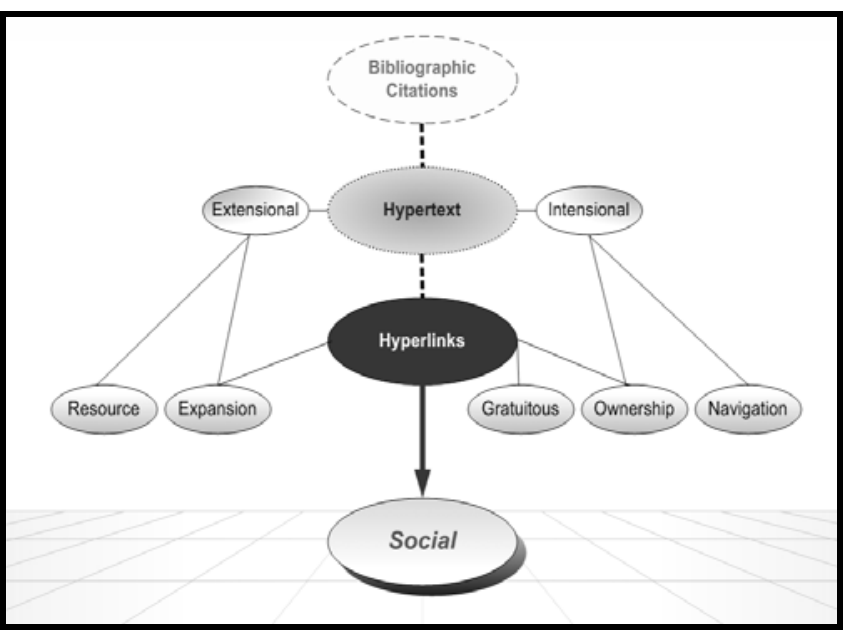

Figure 1: The notion of a social hyperlink

Blogs as publication systems are becoming more democratic, accessible, efficient, and can now even empower users to develop community through electronic social interaction. The development of this type of virtual community is driven by the hyperlink's function as annotation. Hyperlinks become a means for the collaboration and cultivation of community.

\section{Methods}

Extracting our sample from the bloggers who attended the Triangle Blogger Conference held at the University of North Carolina in February 2005, our data collection is on three levels. First, we are particularly interested in the motivations for creating hyperlinks. Each hyperlink on the author's static blog will be analyzed by asking, "Why did you include a hyperlink?” Information will also be recorded about the participant's age, interests, and geographic location. Based on those premises, we anticipate high correlations among geographic locations (because of the nature of our sample) and the interests expressed by the subjects. At the individual level, inquiries about how the blog is used and has developed will be made. On a macro level, participants will be interviewed to discover the various virtual communities they are involved with and how they have seen blog/wiki communities evolve.

\section{Conclusions and Recommendations}

This poster characterizes the reasons for linking using existing taxonomies coupled with the direct approach of interviewing web page authors and their readers. Results from this test are an important substantiation in the debate of relevance in retrieval through which questions about relative importance by linking are answered. The results of the experiments are evaluated to identify a typology of author motivations of hyperlinking. A survey of reader interpretations of the same hyperlinks will be conducted in an identical fashion through a separate study. It is anticipated that these findings will substantiate the function of a hyperlink as an important social dynamic in an evolving Web environment, determined by reader and author associations with independent resources. This research may reveal what shortcomings and gaps exist among motivations and interpretations of hyperlinks.

\section{ACKNOWLEDGMENTS}

This work was partially funded by an unrestricted research gift from Microsoft Research to the Annotation of Structured Data research team in the School of Information and Library Science at the University of North Carolina at Chapel Hill, whose members contributed to this work: Gary Marchionini, Paul Solomon, Catherine Blake, Lili Luo, John MacMullen, Cathy Marshall, Mary Ruvane, David West, and Megan Winget Barret.

The project website is http://ils.unc.edu/annotation

\section{REFERENCES}

Chu, Heting (2005). Taxonomy of inlinked Web entities: What does it imply for Webometric research? Library \& Information Science Research: An International Journal. In press: Available online 28 January 2005.

DeRose, S. J. (1989). Expanding the Notion of Links. Hypertext '89 Proceedings. 249-257.

Haas, Stephanie W. \& Grams, Erika S. (1998). A link taxonomy for Web pages. Proceedings of the 61st Annual Meeting of the American Society for Information Science, 485-495.

Kim, H. J. (2000). Motivations for hyperlinking in scholarly electronic articles: A qualitative study. Journal of the American Society for Information Science, 51(10), 887-899.

Rainie, L. (2005). The State of Blogging. Pew Internet and American Life Project. Retrieved January 14, 2005 from http://www.pewinternet.org/pdfs/PIP_blogging_data.pdf.

Thelwall, M. (2003). What is this link doing here? Beginning a fine-grained process of identifying reasons for academic hyperlink creation. Retrieved February 25, 2005. Information Research, 8(3), http://informationr.net/ir/8-3/paper151.html. 